\title{
Elevated expression of AKT2 correlates with disease severity and poor prognosis in human osteosarcoma
}

\author{
YALONG ZHU* , JIANHUA ZHOU* ${ }^{*}$ YING JI ${ }^{*}$ and BAOQING YU \\ Department of Orthopaedics, Shanghai Pudong Hospital, Fudan University \\ Pudong Medical Center, Shanghai 201399, P.R. China
}

Received September 17, 2013; Accepted April 8, 2014

DOI: $10.3892 / \mathrm{mmr} .2014 .2314$

\begin{abstract}
Members of AKT kinase family are central modulators in numerous signaling cascades, which regulate metabolism, cell proliferation, survival and growth. Previously, the knockdown of AKT2 expression has been demonstrated to enhance the efficacy of chemotherapy in patients with osteosarcoma. However, it is currently unknown whether the aberrant expression of AKT2 has relevance to the progression of osteosarcoma. The aim of the present study was to investigate the clinicopathological and prognostic value of AKT2 in osteosarcoma. Formalin-fixed paraffin embedded osteosarcoma and self-paired non-cancerous bone tissue samples were obtained from 126 patients with osteosarcomas. AKT2 expression was detected by an immunohistochemistry assay. Patient survival rates were determined by the Kaplan-Meier method and log-rank test. Cox regression was adopted for multivariate analysis of the prognostic factors to examine the effect of AKT on event-free survival and overall survival in patients with osteosarcomas. AKT2 expression in osteosarcoma tissues was significantly higher than that in non-cancerous bone tissues (immunostaining score, 6.39 \pm 1.62 vs. $3.46 \pm 1.03 ; \mathrm{P}<0.001)$. In addition, the elevated expression of AKT2 protein was significantly associated with positive recurrence $(\mathrm{P}=0.023)$, the presence of metastasis $(\mathrm{P}=0.006)$ and poor response to chemotherapy $(\mathrm{P}=0.015)$. Furthermore, patients with high AKT2 expression had significantly shorter event-free survival $(\mathrm{P}<0.001)$ and overall survival times $(\mathrm{P}<0.001)$ than those with lower expression levels. Multivariate analysis further demonstrated that AKT2 expression $(\mathrm{P}=0.029$ and 0.016 , respectively), the status of recurrence $(\mathrm{P}=0.018$ and 0.012 , respectively) and metastasis $(\mathrm{P}=0.020$ and 0.015 ,
\end{abstract}

Correspondence to: Professor Baoqing Yu, Department of Orthopaedics, Shanghai Pudong Hospital, Fudan University Pudong Medical Center, 2800 Gongwei Road, Shanghai 201399, P.R. China E-mail: doctorybq@163.com

${ }^{*}$ Contributed equally

Key words: AKT2, osteosarcoma, immunohistochemistry, event-free survival, overall survival respectively), and the response to chemotherapy $(\mathrm{P}=0.011$ and 0.008 , respectively) were all independent prognostic factors for event-free survival and overall survival time. To the best of our knowledge, these data have supported the findings for the first time, that the elevated expression of AKT2 may be associated with aggressive clinical behavior and poor outcome in patients with osteosarcomas. Therefore, AKT2 may be a candidate marker of unfavorable prognosis in osteosarcoma.

\section{Introduction}

Osteosarcoma is one of the most common primary bone malignancies and primarily affects adolescents ( $<20$ years old) (1). It is characterized by frequent vascular invasion, infiltration to adjacent soft tissue, a high rate of local recurrence and early distant metastasis (2). Although multidisciplinary approaches, including chemotherapy and wide resection surgery, are able to significantly improve the clinical outcome for patients with osteosarcoma, the prognosis of advanced cases with distant metastasis and local recurrence remains poor, even with additional extensive chemotherapy (3). Accumulating studies have indicated that a variety of cellular and molecular events may be implicated in the tumorigenesis of osteosarcoma. Therefore, identification of novel markers is required for the prediction of malignant behavior and prognosis of osteosarcomas, in order to guide treatment strategies and improve the clinical outcome of this disease.

AKT family members encode highly similar serine-threonine protein kinases, which belong to the more general class of AGC kinases (related to AMP/GMP kinase and protein kinase C) (4). There are three members of the AKT kinase family: AKT1 (protein kinase B- $\alpha$, PKB- $\alpha$ ), AKT2 (protein kinase B- $\beta$, PKB- $\beta$ ) and AKT3 (protein kinase B- $\gamma$, PKB- $\gamma$ ) (5). All of the AKT kinases lie at the center of the phosphoinositide 3-kinase (PI3k)/AKT signal transduction pathway, which regulates multiple cellular processes, including cell proliferation, growth, survival, transformation and differentiation (6). Activation of the AKT kinases, initiated by extracellular stimuli in a PI3k-dependent manner, regulates downstream signaling proteins that are involved in cell survival, growth, cell cycle progression and apoptotic response (7). Previously, several studies of cancer biology have demonstrated the crucial role of AKT kinases in tumorigenesis and that it may be possible to target them therapeutically and/or as a chemopreventative 
strategy (8). Among the three AKT kinases, AKT2 has been demonstrated to be associated with the development of human cancers. For example, in non-small cell lung cancer, AKT2 contributes to cell survival via different mechanisms (9). In breast cancer cells, ablation of AKT2 may induce cell cycle arrest and be differentially involved in regulating cell migration and the epithelial-to-mesenchymal transition (10). In hepatocellular carcinoma, AKT2 expression may be upregulated and be a novel independent predictor for the development and progression of this malignancy (11). Furthermore, Zhang et al (12) indicated that knockdown of AKT2 expression may have therapeutic applications in enhancing the efficacy of chemotherapy in patients with osteosarcoma. However, it is currently unknown whether the aberrant expression of AKT2 has relevance to the progression of osteosarcoma. The aim of this study was to investigate the clinicopathological and prognostic value of AKT2 in patients with osteosarcoma.

\section{Materials and methods}

Patients and tissue samples. This study was approved by the Research Ethics Committee of Shanghai Pudong Hospital, (Shanghai, China). Written informed consent was obtained from all patients. All specimens were anonymous and handled according to the ethical and legal standards.

Formalin-fixed paraffin embedded osteosarcoma and self-paired non-cancerous bone tissue samples were obtained from 126 patients with osteosarcoma at Shanghai Pudong Hospital between 1998 and 2008. The patients ranged from 12-66 years of age (median, 19 years; mean, 25.2 years). The study cohort included 78 males and 48 females. Clinicopathological parameters, including age, gender, tumor size, tumor site, histological subtype, local recurrence, distant metastasis and response to the neoadjuvant chemotherapy are summarized in Table I. All samples were obtained from primary lesions. The biopsies were performed prior to chemotherapy or radiotherapy for diagnostic purposes.

Follow-up was conducted for all 126 patients with osteosarcoma. Patients were monitored with computed tomography (CT), which was performed every three months during the first three years following chemotherapy, every four months during years four and five, and every six months thereafter. The development of local recurrence and distant metastases were detected by CT scans or magnetic resonance imaging. All cases were independently reviewed by two pathologists and discrepancies resolved by consensus review. Of 126 patients with osteosarcoma, 48 (38.10\%) experienced local recurrence and $52(41.27 \%)$ patients had succumbed to the disease by the last follow-up date. The median follow-up time was 42 months (range, 2-116 months). Overall survival time was calculated from the date of the initial surgical operation to death. Eventfree survival was calculated from the date of the initial surgical operation to the date of secondary cancer, tumor recurrence, distant metastases or mortality from any cause.

Immunohistochemistry analysis. Expression patterns of AKT2 proteins in 126 formalin-fixed paraffin embedded osteosarcomas and self-paired non-cancerous bone tissue samples were detected by immunohistochemical staining. Briefly, all tissue samples were retrieved and divided into $3-\mu \mathrm{m}$ sections and mounted on pre-coated slides. Following deparaffinizing in xylene and washing in a graded series of ethanol, the sections were submerged into EDTA antigenic retrieval buffer (ab93680, Abcam, Cambridge, UK). and microwaved for antigenic retrieval. The slides were incubated with the primary antibody raised against AKT2 (dilution, 1:50, mouse polyclonal antibody; Cell Signaling Technology, Inc., Beverly, MA, USA). All incubations with primary antibody were conducted overnight at $4^{\circ} \mathrm{C}$. Following washing in Tris-buffered saline (Sigma, Munich, Germany), the tissue sections were treated with biotinylated rabbit anti-mouse secondary antibody (Zymed Laboratories, Inc., San Francisco, CA, USA), followed by further incubation with streptavidin-horseradish peroxidase complex (Zymed Laboratories, Inc.). The tissue sections were immersed in 3-amino-9-ethyl carbazole and counterstained with $10 \%$ Mayer's hematoxylin, then dehydrated and mounted in Crystal Mount. Negative control staining was conducted by substituting non-immune rabbit IgG and phosphate-buffered saline for the primary antibodies.

Following hematoxylin counterstaining, immunostaining was scored by two independent observers, who were blinded to the clinicopathological parameters and clinical outcomes of the patients. The scores of the two observers were compared and any discrepancies were trained through re-examining the stainings by the two pathologists to achieve a consensus score. The number of positive-staining cells exhibiting immunoreactivity on the cytoplasm in ten representative microscopic fields was counted and the percentage of positive cells was calculated. The frequency of AKT2 immunoreactivity in the tissue sections was evaluated as negative (0) when no positive cells were observed within the tumor; weak (1) when $<30 \%$ of the tumor cells were positive; moderate (2) when $30-60 \%$ of the tumor cells were positive and strong (3) when $>60 \%$ of tumor cells were positive. The intensity of staining was evaluated as $0,1,2$ and 3 for no staining, weak staining, medium staining and strong staining, respectively. An immunostaining score (IRS) was obtained by multiplying the frequency and intensity score for stained cells (range, 0-9). The cut-off value for IRS of AKT2 protein was selected on the basis of a measure of heterogeneity with the log-rank test statistic, with respect to overall survival. An optimal cut-off value was identified. An IRS of $\geq 4.5$ was used to classify the tumors with high expression and $<4.5$ IRS classified the tumors with low expression of AKT2 protein.

Statistical analysis. SPSS version 16.0 software for Windows (SPSS, Inc., Chicago, IL, USA) and SAS 9.1 (SAS Institute, Cary, NC, USA) were used for statistical analysis. Continuous variables were expressed as the mean \pm standard deviation. Associations between the expression of AKT2 and clinicopathological parameters were assessed using a $\chi^{2}$ test. Patient survival and their differences were determined by Kaplan-Meier method and a log-rank test. Cox regression (proportional hazard model) was adopted for multivariate analysis of prognostic factors. $\mathrm{P}<0.05$ was considered to indicate a statistically significant difference.

\section{Results}

Elevated expression of AKT2 in human osteosarcoma tissues. Expression patterns and subcellular localization of 
Table I. Association between AKT2 expression and different clinicopathological features of osteosarcoma patients.

\begin{tabular}{|c|c|c|c|c|}
\hline \multirow{2}{*}{$\begin{array}{l}\text { Clinicopathological } \\
\text { feature }\end{array}$} & \multirow[b]{2}{*}{ No. of cases } & \multicolumn{2}{|c|}{ AKT2 expression } & \multirow[b]{2}{*}{ P-value } \\
\hline & & $\operatorname{High}(\mathrm{n}, \%)$ & Low $(\mathrm{n}, \%)$ & \\
\hline \multicolumn{5}{|l|}{ Age } \\
\hline$<20$ & 80 & $45(56.25)$ & $35(43.75)$ & \multirow[t]{2}{*}{ NS } \\
\hline$\geq 20$ & 46 & $25(54.35)$ & $21(45.65)$ & \\
\hline \multicolumn{5}{|l|}{ Gender } \\
\hline Male & 82 & $47(57.32)$ & $35(42.68)$ & \multirow[t]{2}{*}{ NS } \\
\hline Female & 44 & $23(52.27)$ & $21(47.73)$ & \\
\hline \multicolumn{5}{|l|}{ Tumor size (cm) } \\
\hline$\geq 5.0$ & 76 & $40(52.63)$ & $36(47.37)$ & \multirow[t]{2}{*}{ NS } \\
\hline$<5.0$ & 50 & $30(60.00)$ & $20(40.00)$ & \\
\hline \multicolumn{5}{|l|}{ Tumor site } \\
\hline Femur & 66 & $37(56.06)$ & $29(43.94)$ & \multirow[t]{3}{*}{ NS } \\
\hline Tibia & 50 & $28(56.00)$ & $22(43.00)$ & \\
\hline Other & 10 & $5(50.00)$ & $5(50.00)$ & \\
\hline \multicolumn{5}{|l|}{ Histological type } \\
\hline Osteoblastic & 58 & $27(46.55)$ & $31(53.45)$ & \multirow[t]{4}{*}{$\mathrm{NS}$} \\
\hline Chondroblastic & 30 & $20(66.67)$ & $10(33.33)$ & \\
\hline Fibroblastic & 22 & $13(59.09)$ & $9(40.91)$ & \\
\hline Other & 16 & $10(62.50)$ & $6(37.50)$ & \\
\hline \multicolumn{5}{|l|}{ Recurrence } \\
\hline Negative & 55 & $22(40.00)$ & $33(60.00)$ & \multirow[t]{2}{*}{0.028} \\
\hline Positive & 71 & $48(67.61)$ & $23(32.39)$ & \\
\hline \multicolumn{5}{|l|}{ Metastasis } \\
\hline Negative & 57 & $18(31.58)$ & $39(68.42)$ & \multirow[t]{2}{*}{0.006} \\
\hline Positive & 69 & $52(75.36)$ & $17(24.64)$ & \\
\hline \multicolumn{5}{|c|}{ Response to chemotherapy } \\
\hline Good & 66 & $28(42.42)$ & $32(57.58)$ & \multirow[t]{2}{*}{0.015} \\
\hline Poor & 60 & $42(70.00)$ & $24(30.00)$ & \\
\hline
\end{tabular}

NS, not significant.

AKT2 protein in 126 pairs of osteosarcoma and matched non-cancerous bone tissues were detected by an immunohistochemical assay. As demonstrated in Fig. 1, strongly positive immunostaining of AKT2 protein was detected in the nucleus and/or cytoplasm of tumor cells in osteosarcoma tissues with a uniform or interspersed granular pattern, while weakly positive or negative immunostaining was detected in non-cancerous bone tissues. In addition, the statistical analysis demonstrated that the expression levels of AKT2 protein in osteosarcoma tissues were significantly higher than in corresponding non-cancerous bone tissues (IRS, $6.39 \pm 1.62$ vs. $3.46 \pm 1.03 ; \mathrm{P}<0.001)$. Of 126 patients with osteosarcomas, 70 $(55.56 \%)$ and $56(44.44 \%)$ belonged to the high and low AKT2 expression groups, respectively.

Elevated expression of AKT2 associates with tumor progression of osteosarcoma tissues. Table I summarizes the association of AKT2 expression with the clinicopathological features of the osteosarcoma patients. The elevated expression of AKT2 protein was significantly associated with positive recurrence $(\mathrm{P}=0.028)$, the presence of metastasis $(\mathrm{P}=0.006)$ and poor response to chemotherapy $(\mathrm{P}=0.015)$. However, the expression of AKT2 protein was not correlated with other factors of patients including age, gender, tumor size, tumor site and histological subtype (all $\mathrm{P}>0.05$ ).

Elevated expression of AKT2 associates with poor prognosis in osteosarcoma patients. The prognostic value of AKT2 expression in human osteosarcoma was further investigated by Kaplan-Meier analysis and a log-rank test. As demonstrated in Fig. 2, osteosarcoma tissues with high AKT2 expression were correlated with a shorter overall survival (Fig. 2A; $\mathrm{P}<0.001$ ) and shorter event-free survival time (Fig. 2B; $\mathrm{P}<0.001$ ). Univariate analysis revealed that the positive recurrence and metastasis, the high expression of AKT2 protein and the poor response to chemotherapy were all significantly correlated with poor overall survival $(\mathrm{P}=0.006, \mathrm{P}<0.001, \mathrm{P}<0.001$ and $\mathrm{P}<0.001$, respectively; Table II) and event-free survival 
Table II. Univariate analysis of prognostic parameters in patients with osteosarcoma by Cox regression analysis.

\begin{tabular}{|c|c|c|c|c|}
\hline \multirow[b]{2}{*}{ Variable } & \multicolumn{2}{|c|}{ Overall survival } & \multicolumn{2}{|c|}{ Event-free survival } \\
\hline & P-value & Relative risk & P-value & Relative risk \\
\hline \multicolumn{5}{|l|}{ Age at diagnosis (years) } \\
\hline$<20$ vs. $\geq 20$ & 0.132 & 0.982 & 0.171 & 1.058 \\
\hline \multicolumn{5}{|l|}{ Gender } \\
\hline Male vs. female & 0.162 & 0.961 & 0.189 & 1.146 \\
\hline \multicolumn{5}{|l|}{ Tumor size (cm) } \\
\hline$<5.0$ vs. $\geq 5.0$ & 0.113 & 1.268 & 0.115 & 1.321 \\
\hline \multicolumn{5}{|l|}{ Tumor site } \\
\hline Femur vs. tibia and other & 0.207 & 1.199 & 0.131 & 1.087 \\
\hline \multicolumn{5}{|l|}{ Histological type } \\
\hline Osteoblastic vs. other & 0.106 & 1.425 & 0.129 & 1.525 \\
\hline \multicolumn{5}{|l|}{ Recurrence } \\
\hline Negative vs. positive & 0.006 & 3.168 & 0.01 & 3.032 \\
\hline \multicolumn{5}{|l|}{ Metastasis } \\
\hline Negative vs. positive & $<0.001$ & 6.879 & $<0.001$ & 6.532 \\
\hline \multicolumn{5}{|l|}{ Response to chemotherapy } \\
\hline Good vs. poor & $<0.001$ & 8.332 & $<0.001$ & 7.969 \\
\hline \multicolumn{5}{|l|}{ AKT2 expression } \\
\hline Low vs. high & $<0.001$ & 6.501 & $<0.001$ & 6.089 \\
\hline
\end{tabular}
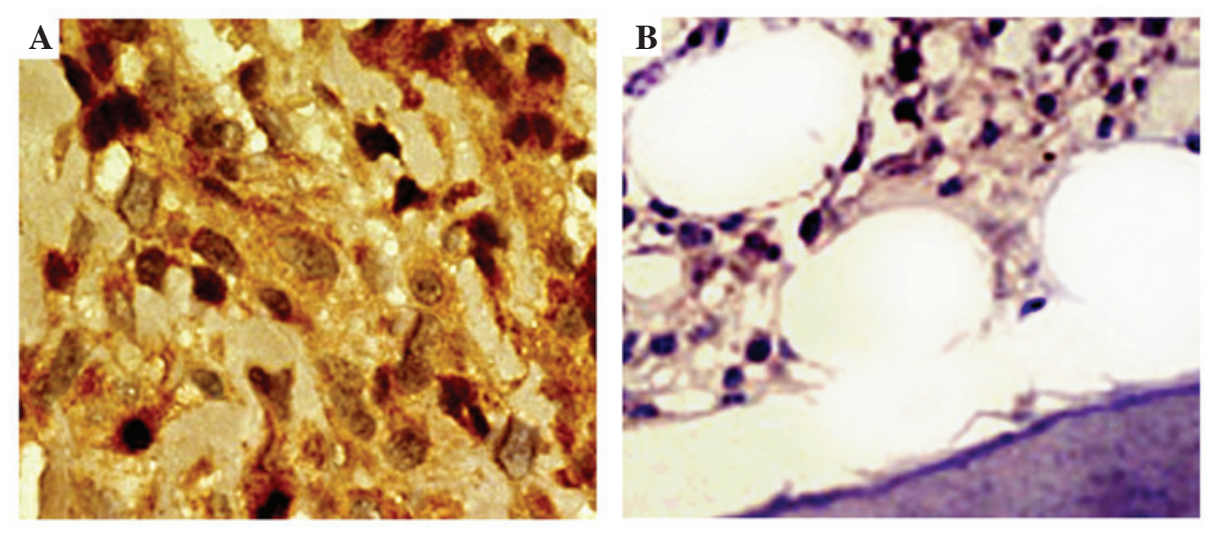

Figure 1. Representative AKT2 protein immunostaining in (A) formalin-fixed paraffin embedded osteosarcoma and (B) self-paired non-cancerous bone tissue samples. Strongly positive immunostaining of AKT2 protein was detected in the nucleus and/or cytoplasm of tumor cells in osteosarcoma tissues with a uniform or interspersed granular pattern, while weakly positive or negative immunostaining were detected in non-cancerous bone tissues (magnification, $\mathrm{x} 400$ ).

$(\mathrm{P}=0.01, \mathrm{P}<0.001, \mathrm{P}<0.001$ and $\mathrm{P}<0.001$, respectively; Table II $)$ of osteosarcoma patients. Multivariate analysis, as summarized in Table III, further demonstrated that AKT2 expression $(\mathrm{P}=0.029$ and 0.016 , respectively), the status of recurrence $(\mathrm{P}=0.018$ and 0.012 , respectively) and metastasis $(\mathrm{P}=0.020$ and 0.015 , respectively), and the response to chemotherapy $(\mathrm{P}=0.011$ and 0.008 , respectively) were all independent prognostic factors for overall survival and event-free survival time.

\section{Discussion}

Despite the advancement of therapeutic strategies, it is extremely difficult to improve the prognosis of patients with osteosarcoma. Furthermore, as $75 \%$ of osteosarcoma cases occur in 10-20-year-old patients, this disease is a significant burden to families and society (13). For these reasons, the screening of efficient molecular markers is of great significance to differentiate the malignant progression, determine the clinicopathological characteristics and predict the clinical outcome of patients with osteosarcoma. In the present study, it was demonstrated that elevated expression of AKT2 may be associated with the progression of osteosarcoma. The results of the immunohistochemical assay reveal that AKT2 is upregulated in primary osteosarcoma specimens. Furthermore, the expression level of AKT2 significantly correlated with aggressive disease severity in the patients. The statistical analyses 
Table III. Multivariate analysis of prognostic parameters in patient with osteosarcomas by Cox regression analysis.

\begin{tabular}{lcccc}
\hline & \multicolumn{2}{c}{ Overall survival } & \multicolumn{2}{c}{ Event-free survival } \\
\cline { 2 - 5 } Variable & P-value & Relative risk & P-value & Relative risk \\
\hline $\begin{array}{l}\text { Recurrence } \\
\text { Negative vs. positive }\end{array}$ & 0.018 & 3.141 & 0.012 & 3.226 \\
$\begin{array}{l}\text { Metastasis } \\
\text { Negative vs. positive }\end{array}$ & 0.020 & 2.817 & 0.015 & 3.962 \\
$\begin{array}{l}\text { Response to chemotherapy } \\
\text { Good vs. poor }\end{array}$ & 0.011 & 3.309 & 0.008 & 4.556 \\
$\begin{array}{l}\text { AKT2 expression } \\
\text { Low vs. high }\end{array}$ & 0.029 & 2.261 & 0.016 & 3.198 \\
\hline
\end{tabular}

A

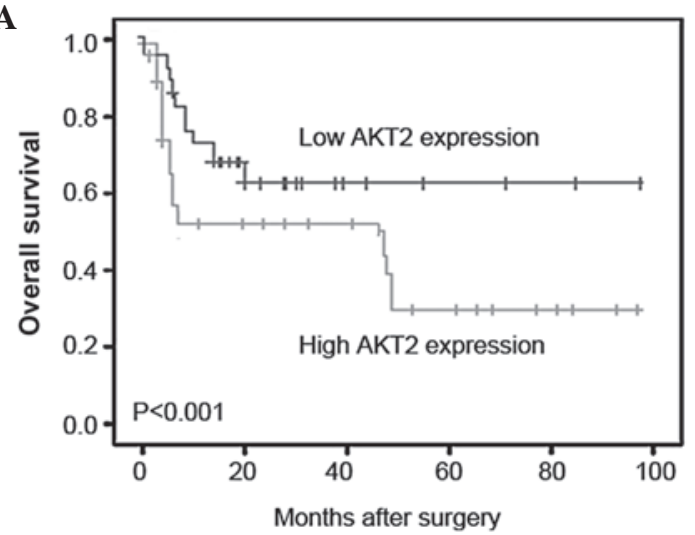

B

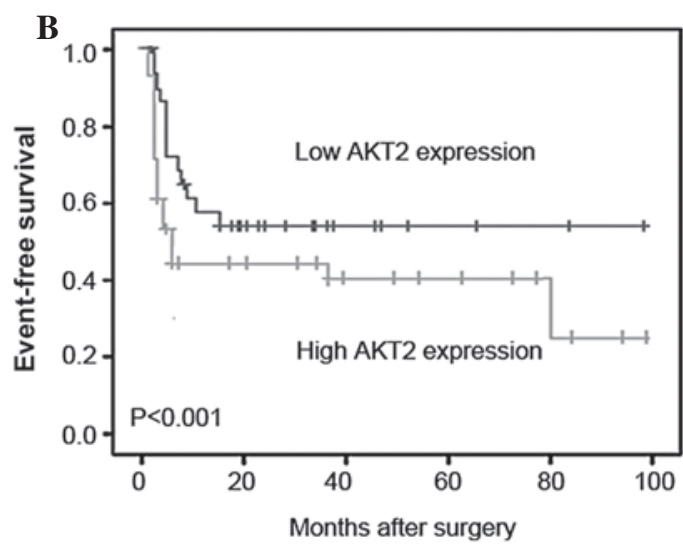

Figure 2. Kaplan-Meier curves for survival time in patients with osteosarcoma are divided according to AKT2 expression. (A) Overall survival and (B) event-free survival of patients with high vs. low AKT2 expression levels are shown. Osteosarcoma patients with high AKT2 expression were correlated with a shorter overall survival $(\mathrm{P}<0.001)$ and shorter event-free survival time $(\mathrm{P}<0.001)$.

revealed that the patients with high expression of AKT2 had a poorer prognosis. Based on these findings, it was suggested that the expression of AKT2 may be of value in predicting the progression and prognosis of patients with osteosarcoma.

Activation of specific AKT family members promotes tumor cell cycling, survival and invasiveness, enhances telomerase activity and modulates angiogenesis (14). These processes are all considered to be hallmarks of cancer. In particular, accumulating evidence indicates that alterations in AKT2 are common in numerous types of human cancer. For example, Cheng et al (15) identified the amplification and overexpression of AKT2 in human ovarian carcinoma tissues and cell lines and Roy et al (16) demonstrated that the overexpression of AKT2 may be an early event during colon tumorigenesis. Furthermore, Cheng et al (17) indicated that the overexpression of AKT2 may be capable of transforming NIH3T3 cells, and AKT2 antisense RNA may inhibit the tumorigeneic phenotype of cancer cells exhibiting amplified AKT2. In the osteosarcoma literature, the activation of AKT2 has been demonstrated to be capable of promoting a chemoresistant phenotype and the inhibition of this kinase may sensitize chemoresistant cancer cells (12). Consistent with these previous findings, data from the present study demonstrated that the expression of AKT2 was significantly increased in osteosarcoma tissues compared with non-cancerous bone tissues. Notably, it was identified that the elevated expression of AKT2 protein may be closely associated with the status of recurrence and metastasis, the response to chemotherapy and the clinical outcome of patients with osteosarcoma, implicating that AKT2 protein may have an oncogenic role during the progression of this malignancy.

In previous years, a variety of mechanisms regarding the oncogenic role of AKT2 have been delineated, but not fully elucidated. It is well established that the activation of AKT kinases is a multi-step process that involves membrane translocation and phosphorylation, and is triggered by the engagement of receptor tyrosine kinases by peptide growth factors and cytokines $(18,19)$. AKT kinases belong to the PI3K/AKT signaling pathway, including the upstream PI3K, PTEN and LKB1, and the downstream tuberous sclerosis complex 2 and eukaryotic initiation factor $4 \mathrm{E}$. All these components have been linked to tumorigenesis (20). The critical step in the signaling cascade leading to AKT activation is stimulation of the growth factor receptor-associated PI3K that forms a direct axis with AKT (21). Aside from this, several serine-threonine phosphatases, including protein phosphatase $2 \mathrm{~A}$, may also be involved in the inactivation of AKT in vivo (22). However, the precise molecular mechanisms for the altered expression of AKT2 in human osteosarcoma remain unclear thus far. 
In conclusion, to the best of our knowledge, this is the first study to indicate that the elevated expression of AKT2 may be associated with aggressive clinical behavior and poor outcome in patients with osteosarcoma. Therefore, AKT2 may be a candidate marker of unfavorable prognosis in osteosarcoma. Further studies of the molecular mechanisms by which AKT2 contributes to the initiation and progression of osteosarcoma are warranted to facilitate further clinical application of this evidence.

\section{References}

1. Dorfman HD and Czerniak B: Bone cancers. Cancer 75 (Supp 1): 203-210, 1995.

2. Jaffe N: Adjuvant chemotherapy in osteosarcoma: an odyssey of rejection and vindication. Cancer Treat Res 152: 219-37, 2009.

3. Kong $\mathrm{C}$ and Hansen MF: Biomarkers in osteosarcoma. Expert Opin Med Diagn 3: 13-23, 2009.

4. Martelli AM, Tabellini G, Bressanin D, Ognibene A, Goto K, Cocco L and Evangelisti C: The emerging multiple roles of nuclear Akt. Biochim Biophys Acta 1823: 2168-2178, 2012.

5. Hemmings BA and Restuccia DF: PI3K-PKB/Akt pathway. Cold Spring Harb Perspect Biol 4: a011189, 2012.

6. Alessi DR and Cohen P: Mechanism of activation and function of protein kinase B. Curr Opin Genet Dev 8: 55-62, 1998.

7. Radisavljevic Z: AKT as locus of cancer positive feedback loops and extreme robustness. J Cell Physiol 228: 522-524, 2013.

8. Burris HA III: Overcoming acquired resistance to anticancer therapy: focus on the PI3K/AKT/mTOR pathway. Cancer Chemother Pharmacol 71: 829-842, 2013.

9. Lee MW, Kim DS, Lee JH, et al: Roles of AKT1 and AKT2 in non-small cell lung cancer cell survival, growth, and migration. Cancer Sci 102: 1822-1828, 2011.

10. Kirkegaard T, Witton CJ, Edwards J, et al: Molecular alterations in AKT1, AKT2 and AKT3 detected in breast and prostatic cancer by FISH. Histopathology 56: 203-211, 2010
11. Xu X, Sakon M, Nagano H, et al: Akt2 expression correlates with prognosis of human hepatocellular carcinoma. Oncol Rep 11: 25-32, 2004.

12. Zhang G, Li M, Zhu X, Bai Y and Yang C: Knockdown of akt sensitizes osteosarcoma cells to apoptosis induced by Cisplatin treatment. Int J Mol Sci 12: 2994-3005, 2011.

13. Chen D, Zhang YJ, Zhu KW and Wang WC: A systematic review of vascular endothelial growth factor expression as a biomarker of prognosis in patients with osteosarcoma. Tumour Biol 34: 1895-1899, 2013.

14. Busaidy NL, Farooki A, Dowlati A, et al: Management of metabolic effects associated with anticancer agents targeting the PI3K-Akt-mTOR pathway. J Clin Oncol 30: 2919-2928, 2012.

15. Cheng JQ, Godwin AK, Bellacosa A, et al: AKT2, a putative oncogene encoding a member of a subfamily of protein-serine/threonine kinases, is amplified in human ovarian carcinomas. Proc Natl Acad Sci USA 89: 9267-9271, 1992.

16. Roy HK, Olusola BF, Clemens DL, Karolski WJ, Ratashak A, Lynch HT and Smyrk TC: AKT proto-oncogene overexpression is an early event during sporadic colon carcinogenesis. Carcinogenesis 23: 201-205, 2002.

17. Cheng JQ, Altomare DA, Klein MA, Lee WC, Kruh GD, Lissy NA and Testa JR: Transforming activity and mitosis-related expression of the AKT2 oncogene: evidence suggesting a link between cell cycle regulation and oncogenesis. Oncogene 14: 2793-2801, 1997.

18. Sheppard K, Kinross KM, Solomon B, Pearson RB and Phillips WA: Targeting PI3 kinase/AKT/mTOR signaling in cancer. Crit Rev Oncog 17: 69-95, 2012.

19. Mahajan K and Mahajan NP: PI3K-independent AKT activation in cancers: a treasure trove for novel therapeutics. J Cell Physiol 227: 3178-3184, 2012.

20. Hafsi S, Pezzino FM, Candido S, et al: Gene alterations in the $\mathrm{PI} 3 \mathrm{~K} / \mathrm{PTEN} / \mathrm{AKT}$ pathway as a mechanism of drug-resistance (review). Int J Oncol 40: 639-644, 2012

21. Toker A: Achieving specificity in Akt signaling in cancer. Adv Biol Regul 52: 78-87, 2012.

22. Shimura T: Acquired radioresistance of cancer and the AKT/GSK3//cyclin D1 overexpression cycle. J Radiat Res 52: $539-544,2011$ 